\title{
A New Step in Excitation-Contraction Coupling
}

IN striated muscle fibres the mechanical response follows the electrical activity of the outer membrane after a very short delay of a few milliseconds. The mechanism connecting the two phenomena has been referred to as excitation-contraction coupling ( $\mathrm{E}-\mathrm{C}$ coupling), an expression coined by Sandow in the early nineteen-fifties. At that time virtually nothing was known about this coupling, except that A. V. Hill had calculated that the diffusion of a hypothetical "activator", liberated by the action potential from the outer membrane, would be much too slow to account for the rapidity of muscle activation.

A great advance towards the understanding of this problem was made when A. F. Huxley and his colleagues provided evidence that the so-called transverse tubules ( $\mathrm{T}$ tubules: more or less radial invaginations of the outer membrane occurring at every sarcomere) could propagate the excitatory signal to the centre of the fibre. The uncoupling between excitation and contraction produced by the selective disruption of these tubules added further support, and the first step of E-C coupling was thus solved.

The later steps are now also reasonably well understood: the use of intracellular indicators for calcium has clearly demonstrated that in response to the excitation, a massive and sudden increase of $\mathrm{Ca}^{2+}$ in the sarcoplasm precedes the onset of the mechanical activity (Jobsis and O'Connor, Biochem. Biophys. Res. Commun, 25, 246 ; 1966 ; Ashley and Ridgway (Nature, 219, 1168 ; 1968). This calcium reacts with the troponin complex located on the thin filaments, relieves the inhibition imposed by this complex on the actin-myosin interaction, and contraction occurs.

But an important question remains to be solved. How does the electrical signal propagated along the $\mathrm{T}$ tubules produce this liberation of calcium? This question is particularly fascinating because of the structural relationships between the $T$ tubules (vector of the excitation) and the longitudinal sarcoplasmic reticulum where enormous amounts of calcium are sequestered in the resting fibre. Electron microscopy has revealed that each $\mathrm{T}$ tubule is in contact with two lateral dilations of the sarcoplasmic reticulum forming a characteristic "triadic" structure which is repeated once or twice every sarcomere. Obviously the "triadic junction" is a key point of the electricalcalcium coupling.

On page 244 of this issue of Nature, Schneider and Chandler report a very interesting observation which seems directly relevant to this question. Using voltageclamp techniques on muscle fibres, these authors discovered that imposed square pulse depolarizations produce a small outwards current, in conditions where all of the time and voltage-dependent changes in sodium and potassium currents are suppressed (using tetrodotoxin, tetraethylammonium and $\mathrm{RbCl}$ ). This current decays with time. At the end of the pulse, an inwards current can be detected. The interesting point is that the total amount of charge involved in the "on" and "off" responses is identical and remains so in various experimental conditions.

Schneider and Chandler suggest that the simplest inter- pretation of this relation is that fixed charges, located in or near a structure sensitive to the polarization, move reversibly in response to changes in polarization. This view is supported by the relation between the amount of charge which moves and the membrane potential. Practically no signal is detected when the holding potential is $-100 \mathrm{mV}$ or more negative. For less negative potentials, the amount of charge increases with depolarization following an S-shaped curve, with a mid-point around $-50 \mathrm{mV}$ and a maximum value around $-20 \mathrm{mV}$. These figures are illuminating, for the movement of charge occurs in a range of potentials where the mechanical response of the muscle is critically dependent on the membrane polarization. This strongly suggests a connexion with the $\mathrm{E}-\mathrm{C}$ coupling.

The nature of these charges and their location are unknown. Nevertheless, the speculation presented by the authors at the end of their article is very interesting because they interpret their observation in connexion with the fine structure of the triadic junction. Electron microscope studies of Franzini-Armstrong (J. Cell. Biol., 47, 488; 1970) have revealed that the mode of contact of the $T$ tubules with the sacroplasmic reticulum is unique among the various types of membrane contacts. The two structures are connected through dense projections ("feet") coming from the terminal cisternae of the sarcoplasmic reticulum, and the space between these feet is in free communication with the sarcoplasm. The density of feet is about 700 per $\mu \mathrm{m}^{2}$ of tubule membrane. By comparison, Schneider and Chandler estimate from their results that the density of charge groups is around 300 per $\mu \mathrm{m}^{2}$, assuming that they are uniformly distributed on the outer and the $\mathrm{T}$ tubule membranes. Though the agreement of these densities might be quite fortuitous, it provides the highly stimulating hypothesis that the excitatory signal is passing from the $T$ tubule to the sarcoplasmic reticulum membrane by way of charges moving in the connecting feet, and Schneider and Chandler have found how to follow the trace of this passage. If true, this is progress.

It remains to be solved how this possible charge transfer across the feet triggers the release of calcium. Schneider and Chandler cautiously suggest that the release is somehow a function of the quantity of charge involved, but the speculation could be pushed a little further. It is probable that the massive and sudden release of calcium produced by the excitation is caused by a permeability change of a large fraction of the sarcoplasmic reticulum membrane (or at least of the terminal cisternae). But the measurements of Franzini-Armstrong have shown that only a few per cent of the sarcoplasmic reticulum membrane are involved in the feet connexion. Therefore, the signal passing through the feet should somehow be "propagated" or "amplified" in order to influence large surfaces of the sarcoplasmic reticulum. In this context, it would be very interesting to see whether a suitable mechanism could be provided by the property of autoamplification of the release of calcium itself, discovered by Ford and Podolsky (Science, 167, 58 ; 1970) and by Endo (Nature, 228, 34 ; 1970) and his colleagues. 